\title{
Diffusion of small light particles in a solvent of large massive molecules
}

\author{
Rajesh K. Murarka, Sarika Bhattacharyya, ${ }^{\text {a) }}$ and Biman Bagchib) \\ Solid State and Structural Chemistry Unit, Indian Institute of Science, Bangalore, India 560012
}

\begin{abstract}
We study the diffusion of small light particles in a solvent which consists of large heavy particles. The intermolecular interactions are chosen to approximately mimic a water-sucrose (or waterpolysaccharide) mixture. Both computer simulation and mode coupling theoretical (MCT) calculations have been performed for a solvent-to-solute size ratio 5 and for a large variation of the mass ratio, keeping the mass of the solute fixed. Even in the limit of large mass ratio the solute motion is found to remain surprisingly coupled to the solvent dynamics. Interestingly, at intermediate values of the mass ratio, the self-intermediate scattering function of the solute, $F_{s}(k, t)$ (where $k$ is the wave number and $t$ is the time), develops a stretching at long time which could be fitted to a stretched exponential function with a $k$-dependent exponent, $\beta$. For very large mass ratio, we find the existence of two stretched exponentials separated by a power law type plateau. The analysis of the trajectory shows the coexistence of both hopping and continuous motions for both the solute and the solvent particles. It is found that for mass ratio 5, the MCT calculations of the self-diffusion underestimates the simulated value by about $20 \%$, which appears to be reasonable because the conventional form of the MCT does not include the hopping mode. However, for larger mass ratio, MCT appears to breakdown more severely. The breakdown of the MCT for large mass ratio can be connected to a similar breakdown near the glass transition.
\end{abstract}

\section{INTRODUCTION}

The issue of diffusion of small light particles in a solvent composed of larger and heavier particles is unconventional because the role of the solvent in the solute diffusion is different from the case where the sizes are comparable. There are two limits that can be identified for such systems. One limit is the well studied Lorentz gas system, which consists of a single point particle moving in a triangular array of immobile disk scatters. Here the motion of the point particle can be modelled by random walk between traps. ${ }^{1-3}$ The other limit is where the size of the solute particle is still smaller than that of the solvent molecules but it has a finite size (that is, not a point) and while the solvent is slow (compared to the solute particles) but not completely immobile. In the latter case, the translational diffusion of the solute is often attempted to describe the well known hydrodynamic StokesEinstein (SE) relation given by, ${ }^{4,5}$

$$
D=\frac{k_{B} T}{C \eta R}
$$

where $k_{B}$ is the Boltzmann constant, $T$ is the absolute temperature, $C$ is a numerical constant determined by the hydrodynamic boundary condition, $\eta$ is the shear viscosity of the solvent, and $R$ is the radius of the diffusing particle. The validity of Eq. (1) for small solutes is, of course, questionable. $^{4-6}$

\footnotetext{
a) Present address: Arthur Amos Noyes Laboratory of Chemical Physics, California Institute of Technology, Pasadena, California 91125.

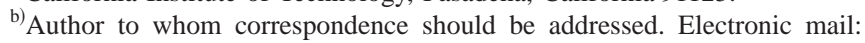
bbagchi@sscu.iisc.ernet.in
}

There have been many experimental, ${ }^{6,7}$ computer simulation, ${ }^{8-10}$ and theoretical ${ }^{11,12}$ studies of diffusion of small solute particles in a solvent composed of larger particles. All these studies show that the SE relation significantly underestimates the diffusion coefficient. To explain the enhanced diffusion, sometimes an empirical modification of the SE relation is used. ${ }^{6,7}$ It is considered that $D \propto \eta^{-\alpha}$, where $\alpha \simeq 2 / 3$. This fractional viscosity dependence is referred to as the microviscosity effect which implies that the viscosity around the small solute is rather different from that of the bulk viscosity. The enhanced diffusion value has also been explained in terms of the effective hydrodynamic radius. ${ }^{4,13}$

The earlier mode coupling theoretical (MCT) studies ${ }^{11,12}$ of diffusion of smaller solutes in a solvent composed of larger size molecules attributed the enhanced diffusion to the decoupling of the solute motion from the structural relaxation of the solvent. The MCT studies suggest that this decoupling of the solute motion from the structural relaxation of the solvent can lead to the fractional viscosity dependence often observed in supercooled liquids.

However, there have been no systematic studies of the effects of the variation of size and mass of the solute-solvent system. In this article we have explored the diffusional mechanism of the isolated small particles (solute) in a liquid composed of larger particles (solvent), both analytically and numerically. The study is performed by keeping the solventto-solute size ratio $\left(S_{R}\right)$ fixed at 5 , but varying the mass of the solvent over a large range, by keeping the mass of the solute fixed. That is, the mass ratio $M_{R}$ (solvent mass/solute mass) is progressively raised to higher values. This system is 
expected to mimic some aspects of the water-sucrose or water-polysaccharide mixtures. ${ }^{14}$

The trajectories of the solute and the solvent show the coexistence of both hopping and continuous motions. As the solvent mass is increased, the self-intermediate scattering function of the solute develops an interesting stretch for a long time. For the larger mass ratio, we see the existence of two stretched exponentials separated by a power law type plateau.

The mode coupling theory calculation of the selfdiffusion coefficient of solute particles performed in the limit of small mass ratio (of 5) is found to be in qualitative agreement with the simulated diffusion-MCT underestimates the diffusion by about $20 \%$. Thus, although the MCT underestimates the diffusion, the agreement is satisfactory in light of the contribution from the hopping mode to diffusion which MCT does not explicitly take into account. However, the deviation from the simulated value increases with an increase in mass ratio (which is equivalent to the increase of the mass of the solvent particles). In the limit of large mass ratio, MCT breaks down. The binary contribution to the total friction is found to decrease as one increases the mass of the solvent. In addition, due to the development of stretching in the self-intermediate scattering function of the solute and the inherent slow solvent dynamics, there remains a strong coupling of the solute motion to the solvent density mode. This enhanced coupling at larger mass ratio came as a surprise to us.

In the limit of very large mass ratio, the motion of the light solute particle resembles that of its motion in an almost frozen disorder system like near the glass transition temperature. Thus, the breakdown of MCT in the limit of large mass ratio could be connected to its failure near the glass transition temperature. Of course, one should note that in the limit of mass of the solvent goes to infinity, the basic assumption of conventional MCT breaks down.

It is widely believed that in a deeply supercooled liquid close to its glass transition temperature $\left(T_{g}\right)$, the hopping mode is the dominant mode in the system which controls the mass transport and the stress relaxation. Recently, a computer simulation study of a deeply supercooled binary mixture $^{15}$ has shown evidence of an intimate connection between the anisotropy in local stress and the particle hopping. It was shown that the local anisotropy in the stress is responsible for the particle hopping in a particular direction. Furthermore, it was suggested that the local frustration present in the system (which is more in a binary mixture with components of different sizes) could cause the local anisotropy in the stress which in turn acts as a driving force for hopping. However, in the present study, the density (or the pressure) of the system is not as high as that of a deeply supercooled system. The relaxation of the stress is found to occur much faster and it relaxes almost completely within our simulation time window even for the largest mass ratio. Consequently, the microscopic origin of particle hopping here could be different than that for a deeply supercooled liquid.

The layout of the rest of the paper is as follows: Section II deals with the system and simulation details. The simulation results and discussions are given in Sec. III, and the mode coupling theoretical analysis is presented in Sec. IV. In Sec. V, we discuss the possible effect of heterogeneity probed by the solute on the self-intermediate scattering function of the solute. Finally, in Sec. VI we present the conclusions of the study.

\section{SYSTEM AND SIMULATION DETAILS}

We performed a series of equilibrium isothermalisobaric ensemble (NPT) molecular dynamics (MD) simulation of binary mixtures in three dimensions for an infinitesimal small value of the mole fraction of one of the species. The binary system studied here contains a total of $N=500$ particles consisting of two species of particles, with $N_{1}$ $=490$ and $N_{2}=10$ number of particles. Hereafter, we refer to indices 1 and 2, respectively, for the solvent and solute particles. Thus, the mixture under study contains $2 \%$ of the solute particles. The interaction between any two particles is modeled by means of the shifted force Lennard-Jones (LJ) pair potential, ${ }^{16}$ where the standard $\mathrm{LJ}$ is given by

$$
u_{i j}^{\mathrm{LJ}}=4 \epsilon_{i j}\left[\left(\frac{\sigma_{i j}}{r_{i j}}\right)^{12}-\left(\frac{\sigma_{i j}}{r_{i j}}\right)^{6}\right],
$$

where $i$ and $j$ denote two different particles (1 and 2). In our model system, the potential parameters are as follows: $\epsilon_{11}$ $=1.0, \sigma_{11}=1.0, \epsilon_{22}=1.0, \sigma_{22}=0.2, \epsilon_{12}=2.0$ (enhanced attraction), and $\sigma_{12}=0.6$. The mass of the solute particles is chosen to be $m_{2}=0.2$ where the solvent (species 1) mass $m_{1}$ is increasingly varied and four different values are chosen 1 , 5,10 , and 50 . Thus, in this study we examined four different solvent-to-solute mass ratios, $M_{R}=m_{1} / m_{2}=5,25,50$, and 250 for a fixed solvent-to-solute size ratio, $S_{R}=\sigma_{11} / \sigma_{22}$ $=5$. Note that in the model system being studied the solutesolvent interaction $\left(\epsilon_{12}\right)$ is much stronger than both of their respective pure counterparts. In order to lower the computational burden the potential has been truncated with a cutoff radius of $2.5 \sigma_{11}$. All the quantities in this study are given in reduced units, that is, length in units of $\sigma_{11}$, temperature $T$ in units of $\epsilon_{11} / k_{B}$, pressure $P$ in units of $\epsilon_{11} / \sigma_{11}^{3}$, and the mass in units of $m$, which can be assumed as an argon (Ar) mass unit. The corresponding microscopic time scale is $\tau$ $=\sqrt{m \sigma_{11}^{2} / \epsilon_{11}}$.

All simulations in the NPT ensemble were performed using the Nosé-Hoover-Andersen method, ${ }^{17}$ where the external reduced temperature is held fixed at $T^{*}=0.8$. The external reduced pressure has been kept fixed at $P^{*}=6.0$. The reduced average density $\bar{\rho}^{*}$ of the system corresponding to this thermodynamic state point is 0.989 for all the mass ratios being studied. Throughout the course of the simulations, the barostat and the system's degrees of freedom are coupled to an independent Nosé-Hoover chain ${ }^{18}$ (NHC) of thermostats, each of length 5 . The extended system equations of motion are integrated using the reversible integrator method $^{19}$ with a small time step of 0.0002 . The higher order multiple time step method ${ }^{20}$ has been employed in the NHC evolution operator which lead to stable energy conservation for non-Hamiltonian dynamical systems. ${ }^{21}$ The extended system time scale parameter used in the calculations was taken to be 0.9274 for both the barostat and thermostats. 


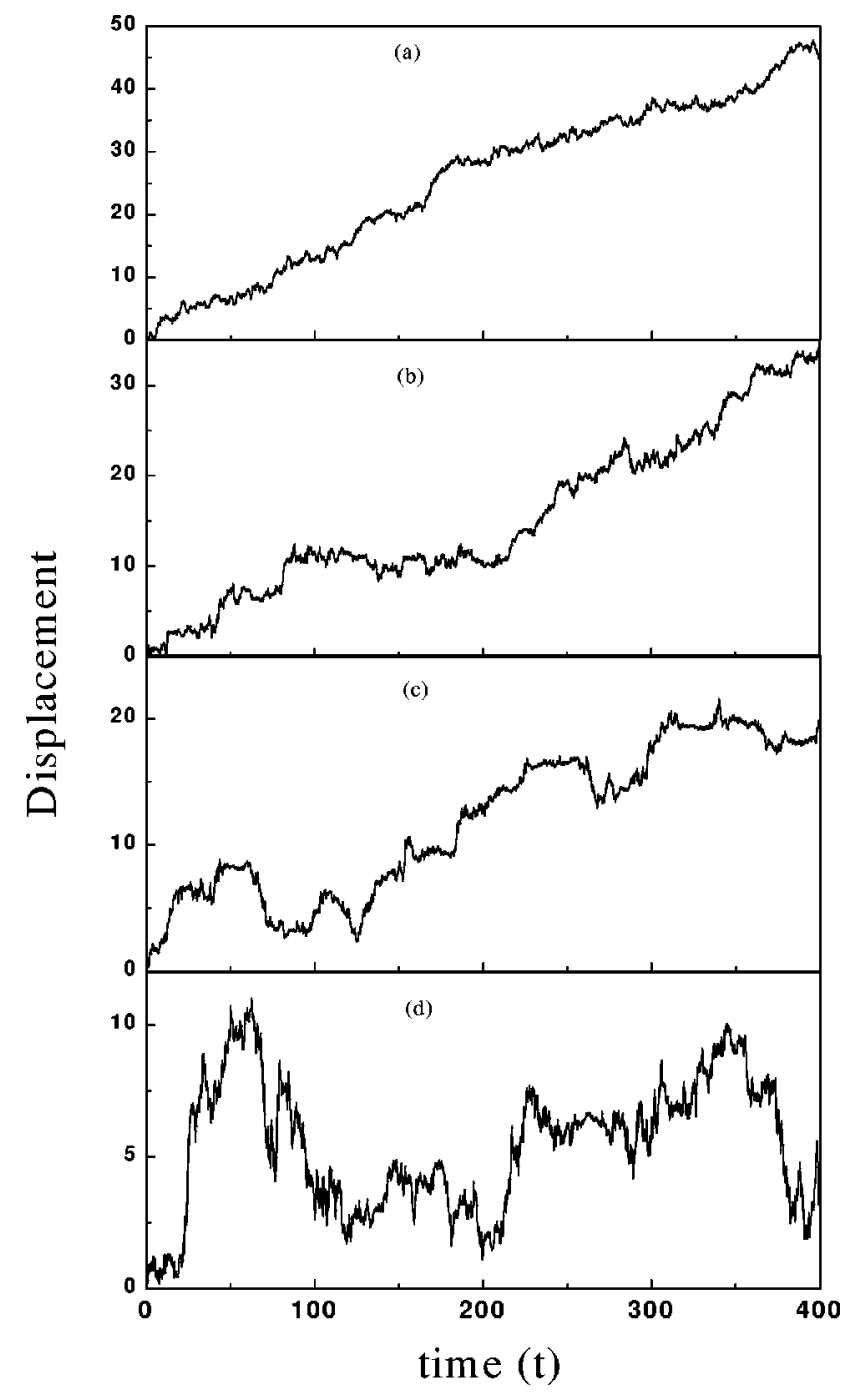

FIG. 1. The time dependence of the displacements for a solute particle at different solvent-to-solute mass ratio, $M_{R}$ : (a) $M_{R}=5$, (b) $M_{R}=25$, (c) $M_{R}=50$, and (d) $M_{R}=250$. Note that the time is scaled by $\sqrt{m \sigma_{11}^{2} / k_{B} T}$. The time unit is equal to $2.2 \mathrm{ps}$ if argon units are assumed.

The systems were equilibrated for $10^{6}$ time steps and simulations were carried out for another $2.0 \times 10^{6}$ production steps following equilibration, during which the quantities of interest are calculated. The dynamic quantities are averaged over three such independent runs for better improvement of the statistics. We have also calculated the partial radial distribution functions $\left[g_{11}(r)\right.$ and $\left.g_{12}(r)\right]$ to make sure that there is no crystallization.

\section{SIMULATION RESULTS AND DISCUSSION}

In Fig. 1 we show typical solute trajectories for four different solvent-to-solute mass ratios, $M_{R}\left(=m_{1} / m_{2}, m_{1}\right.$ is the mass of the solvent particles). The trajectories reveal an interesting dependence on $M_{R}$. At the value of $M_{R}$ equal to 5 , the solute trajectory is mostly continuous with occasional hops. As the mass ratio $M_{R}$ is increased, the solute motion gets more trapped, and its motion tends to become discontinuous where displacements occur mostly by hopping. This is because with an increase in the solvent mass the time scale of motion of the solvent particles become increasingly

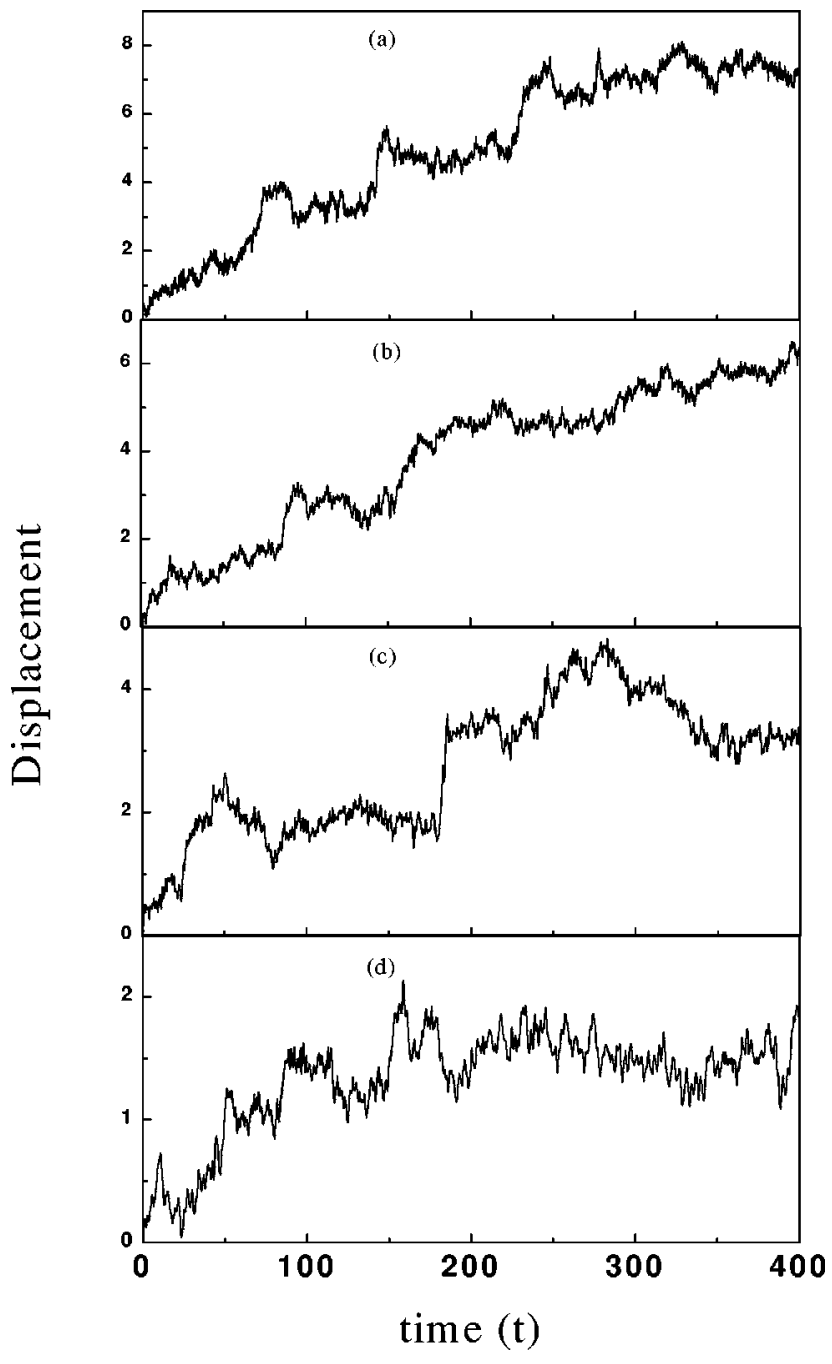

FIG. 2. The same plot as in Fig. 1, but for a solvent particle at different $M_{R}$ : (a) $M_{R}=5$, (b) $M_{R}=25$, (c) $M_{R}=50$, and (d) $M_{R}=250$. Note that here also the time is scaled by $\sqrt{m \sigma_{11}^{2} / k_{B} T}$.

slower. Thus, the solute gets caged by the solvent particles and keeps rattling between a cage-until one solvent particle moves considerably to disperse the solute trajectory (see trajectory for $M_{R}=250$ ). Thus, there is a remarkable change in the solute's motion in going from $M_{R}=5$ [Fig. 1(a)] to $M_{R}$ $=250$ [Fig. 1(d)].

In Fig. 2 we plot the solvent trajectories for the different solvent-to-solute mass ratio, $M_{R}$. We find that for all values of $M_{R}$, there is a coexistence of hopping and continuous motion of the solvent molecules. At higher solvent mass, as expected, the magnitude of displacement becomes less and hopping becomes less frequent, but, surprisingly, the jump motion still persists.

Figure 3 displays the decay behavior of the selfintermediate scattering function $\left[F_{s}(k, t)\right]$ of the solute for a different mass ratio $M_{R}$, at reduced wave number $k^{*}$ $=k \sigma_{11} \sim 2 \pi$. The plot shows that $F_{s}(k, t)$ begins to stretch more for higher solvent mass. This stretching of $F_{s}(k, t)$ is kind of novel and we have examined it in detail.

After the initial Gaussian decay, $F_{s}(k, t)$, for smaller values of $M_{R}$, can be fitted to a single stretched exponential where the exponent $\beta \simeq 0.6$. However, for higher mass of the 


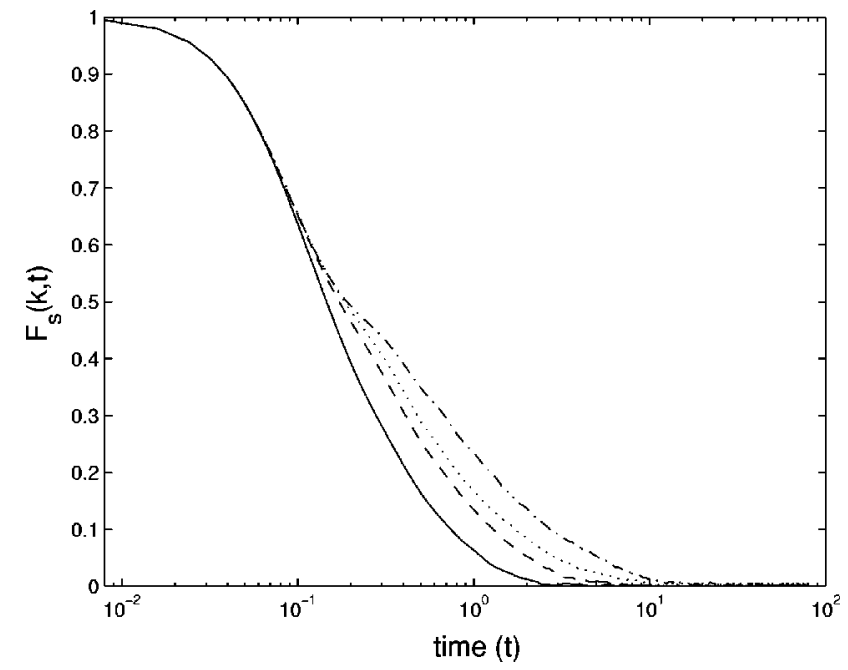

FIG. 3. The self-intermediate scattering function $F_{s}(k, t)$ for the solute particles for different mass ratio $M_{R}$, at reduced wave number $k^{*}=k \sigma_{11}$ $\sim 2 \pi$. The solid line represents $M_{R}=5$, the dashed line is for $M_{R}=25$, the dotted line is for $M_{R}=50$, and the dot-dashed line is for $M_{R}=250$. Note the stretching in $F_{s}(k, t)$ at longer time with an increase in $M_{R}$. For details, see the text.

solvent, $F_{s}(k, t)$ can be fitted only to a sum of two stretched exponentials.

The behavior of $F_{s}(k, t)$ of the solvent for all the solvent masses studied is shown in Fig. 4. The plot shows that (as expected) the time scale of relaxation of $F_{s}(k, t)$ becomes longer as the solvent mass is increased. However, the selfintermediate scattering function of the solvent does not display any stretching at long times, even for the largest mass ratio considered. The decay can be fitted by sum of a shorttime Gaussian and a long-time exponential function.

The reason that $F_{s}(k, t)$ of the solute shows such stretching but that of the solvent does not, can be explained as follows. Due to the small size and the lighter weight of the solute, the time scale of motion of the solute particles is

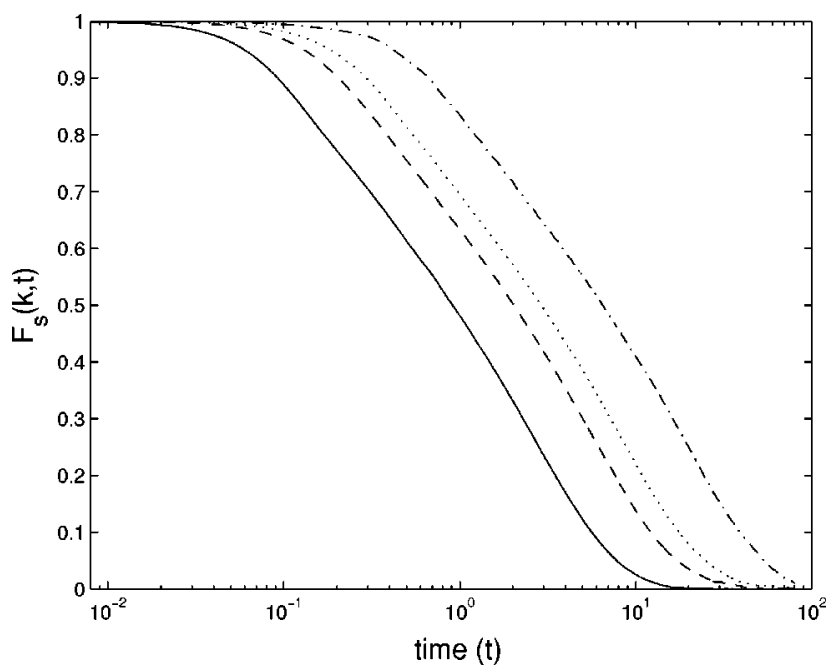

FIG. 4. The self-intermediate scattering function $F_{s}(k, t)$ for the solvent particles for different mass ratio $M_{R}$, at reduced wave number $k^{*} \sim 2 \pi$. The solid line represents $M_{R}=5$, the dashed line is for $M_{R}=25$, the dotted line is for $M_{R}=50$, and the dot-dashed line is for $M_{R}=250$.

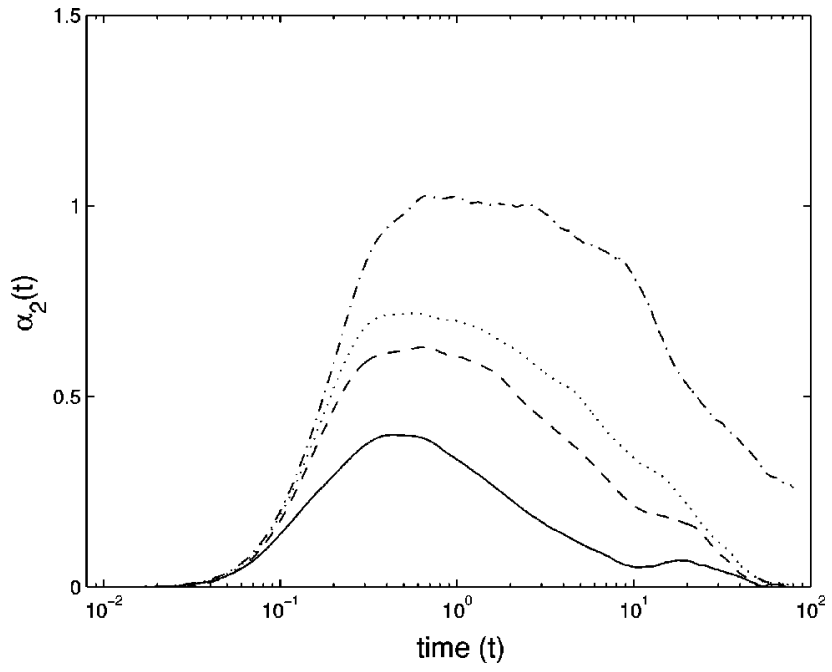

FIG. 5. The behavior of the non-Gaussian parameter $\alpha_{2}(t)$ calculated for the solute particles at different mass ratio $M_{R}$. The solid line, the dashed line, the dotted line, and the dot-dashed line are for $M_{R}=5,25,50$, and 250, respectively.

much shorter compared to that of the solvent particles. Consequently, the solute motion probes more heterogeneity during the time scale of decay of $F_{s}(k, t)$ of the solute. This heterogeneity probed by the solute increases as the solvent mass is increased. Since the solvent motion is much slower, it probes enough configurations during the time scale of decay of its $F_{s}(k, t)$.

In order to quantify the degree of heterogeneity probed by the solute, we have plotted the non-Gaussian parameter $\alpha_{2}(t),{ }^{22}$ for the solute, in Fig. 5. Clearly, the heterogeneity probed by the solute [quantified by the peak height of $\alpha_{2}(t)$ ] increases as the solvent mass is increased. On the other hand, $\alpha_{2}(t)$ of the solvent shows no such increase in the peak height of $\alpha_{2}(t)$ which remains small and unaltered, although the position of the peak shifts to longer time as the mass of the solvent is increased. We have discussed this analysis in more detail in Sec. V.

The role of the local heterogeneity can be further explored by calculating $F_{s}(k, t)$ at a wave number corresponding to solute-solvent average separation. This corresponds to $k^{*}=2 \pi / \sigma_{12}$, where $\sigma_{12}=\frac{1}{2}\left(\sigma_{1}+\sigma_{2}\right)$. In Fig. 6 we plot the self-dynamic structure factor of the solute for the different mass ratio $M_{R}$, at a reduced wave number $k^{*} \sim 2 \pi / \sigma_{12}$. This is primarily the wave number probed by the solute. At this wave number one observes more stretching of $F_{s}(k, t)$ at longer times, for all the mass ratios. This is in agreement with the above argument that since the time window probed by the solute is smaller at higher $k$, it probes even larger heterogeneity.

The emergence of the plateau between the two stretched exponentials in $F_{s}(k, t)$ of the solute (Fig. 6), can be attributed to the separation of the time scale of the binary collision and the solvent density mode contribution to the particle motion. This separation of time scale increases as the solvent mass is increased. As the decay after the plateau is mainly due to the density mode contribution, the plateau becomes more prominent as the mass of the solvent is increased. 


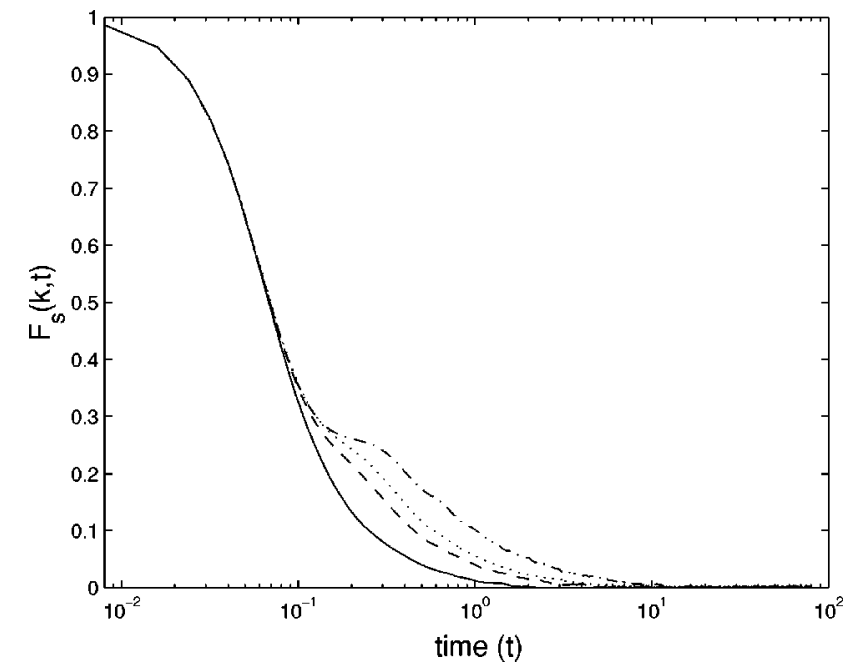

FIG. 6. The self-intermediate scattering function $F_{s}(k, t)$ for the solute particles for different mass ratio $M_{R}$ as in Fig. 3, but at the reduced wave number $k^{*} \sim 2 \pi / \sigma_{12}$. This is primarily the wave number probed by the solute. Note the emergence of a plateau at larger mass ratio. For details, see the text.

Table I clearly shows the separation of time scales where the value of the time constants and the exponents obtained from the two different stretched exponential fits to $F_{s}(k, t)$ are presented for different mass ratio $M_{R}$.

The $F_{s}(k, t)$ of each of the individual solute particle obtained from a single MD run is shown in Fig. 7, at $k^{*}$ $\sim 2 \pi / \sigma_{12}$ and for $M_{R}=250$. We find that not only all of them have a different time scale of relaxation but each of them shows considerable stretching (the exponent $\beta \simeq 0.6$ ) at a longer time. This confirms further that each of the solute particles probe the heterogeneous structure and dynamics of the solvent.

In Table II we present the scaled average (over all the solute particles and three independent MD runs) diffusion value of the solute particles obtained from the slope of the mean square displacement (MSD) in the diffusive limit, for different mass ratio, $M_{R}$. The values of the solute diffusion decreases as the mass of the solvent is increased, as expected. The mass dependence can be fitted to a power law as clearly manifested in Fig. 8 where we have plotted $\ln 1 / D_{2}$ against $\ln m_{1} / m_{2}$, where $D_{2}$ is the self-diffusion of the solute particles. The slope of the line is about 0.13 . The small value of the exponent is clearly an indication of the weak mass dependence of the self-diffusion coefficient of the small solute particle on the mass of the bigger solvent particles.

TABLE I. The time constants $\left(\tau_{1}\right.$ and $\left.\tau_{2}\right)$ and the exponents $\left(\beta_{1}\right.$ and $\left.\beta_{2}\right)$ obtained from the stretched exponential fit to the $F_{s}(k, t)$ at the reduced wave number $k^{*} \sim 2 \pi / \sigma_{12}$ for different solvent-to-solute mass ratio $\left(M_{R}\right)$.

\begin{tabular}{rllll}
\hline \hline $\mathbf{M}_{\mathbf{R}}=\frac{\mathbf{m}_{\mathbf{1}}}{\mathbf{m}_{\mathbf{2}}}$ & \multicolumn{1}{l}{$\tau_{1}$} & $\beta_{1}$ & $\tau_{2}$ & $\beta_{2}$ \\
\hline 5 & 0.082 & 0.70 & & \\
25 & 0.08 & 0.96 & 0.49 & 0.67 \\
50 & 0.083 & 0.94 & 0.59 & 0.64 \\
250 & 0.085 & 0.91 & 1.01 & 0.635 \\
\hline \hline
\end{tabular}

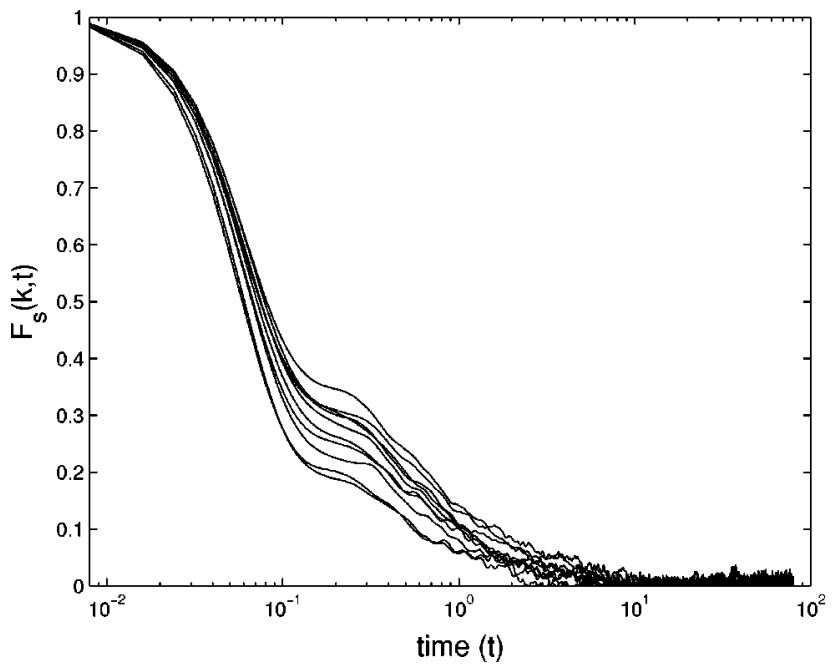

FIG. 7. The self-intermediate scattering function $F_{s}(k, t)$ for each of the ten individual solute particle obtained from a single MD run for mass ratio $M_{R}=250$. They are calculated at the reduced wave number $k^{*} \sim 2 \pi / \sigma_{12}$.

Interestingly, it is to be noted that a similar weak power law mass dependence was seen in the self-diffusion coefficient of a tagged particle on its mass - the exponent was often found to be around 0.1. Recently, a self-consistent mode coupling theory (MCT) analysis successfully explained this weak mass dependence. ${ }^{23}$

In Fig. 9 we plot the normalized velocity autocorrelation function $C_{v}(t)$ of the solute particles for the different values of $M_{R}$. The velocity correlation function shows highly interesting features at larger mass ratio. Not only does the negative dip becomes larger, but there develops a second minimum or an extended negative plateau which becomes prominent as the mass of the solvent is increased. Interestingly, as can be seen from the figure the $C_{v}(t)$ shows an oscillatory behavior that persists for a long period. This is clear evidence for the "dynamic cage" formation in which the solute particle is seen to execute a damped oscillatory motion. Because of the increasing effective structural rigidity of the neighboring solvent particles as the mass of the solvent increases, the motion of the solute particle can be modelled as a damped oscillator which is reminiscent of the behavior observed in a deeply supercooled liquid near the glass transition temperature. ${ }^{24}$

The understanding of the microscopic origin of the development of an increasingly negative dip followed by pronounced oscillations at longer times in the velocity autocorrelation function of a supercooled liquid is the subject of

TABLE II. The self-diffusion coefficient values of the solute particle predicted by the simulation and obtained from the MCT calculations for different solvent-to-solute mass ratio $\left(M_{R}\right)$.

\begin{tabular}{rll}
\hline \hline $\mathbf{M}_{\mathbf{R}}=\frac{\mathbf{m}_{\mathbf{1}}}{\mathbf{m}_{\mathbf{2}}}$ & $\mathbf{D}_{\mathbf{2}}^{\text {sim }}$ & $\mathbf{D}_{\mathbf{2}}^{\mathbf{M C T}}$ \\
\hline 5 & 0.135 & 0.1065 \\
25 & 0.108 & 0.0675 \\
50 & 0.101 & 0.053 \\
250 & 0.0805 & 0.032 \\
\hline \hline
\end{tabular}




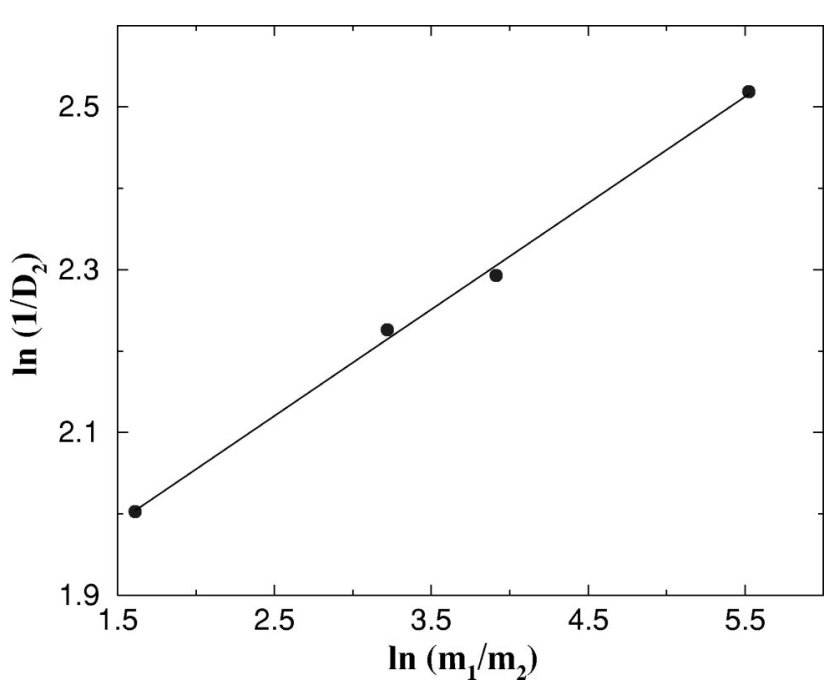

FIG. 8. The plot of $\ln _{e} 1 / D_{2}$ vs $\ln _{e} m_{1} / m_{2}$, where $D_{2}$ is the self-diffusion of the solute. $m_{1}$ and $m_{2}$ are the masses of the solvent and solute particles, respectively. The slope of the straight line is about 0.13 . This suggests a weak power-law mass dependence of the solute diffusion on the mass of the bigger solvent particles.

much current interest. The novel molecular-dynamics simulation study of Kivelson and co-workers ${ }^{25}$ surely provide a step forward in this direction. Their simulation study had shown that the single particle velocity autocorrelation function could be thought of as a sum of the local rattling motion relative to the center-of-mass of the neighboring cluster and the motion of the center-of-mass of that cluster. Furthermore, it was observed that the rich structure displayed by the velocity autocorrelation function at high density arise primarily from the relative motion of the tagged particle, that is, the rattling motion within the cage formed by the neighboring particles.

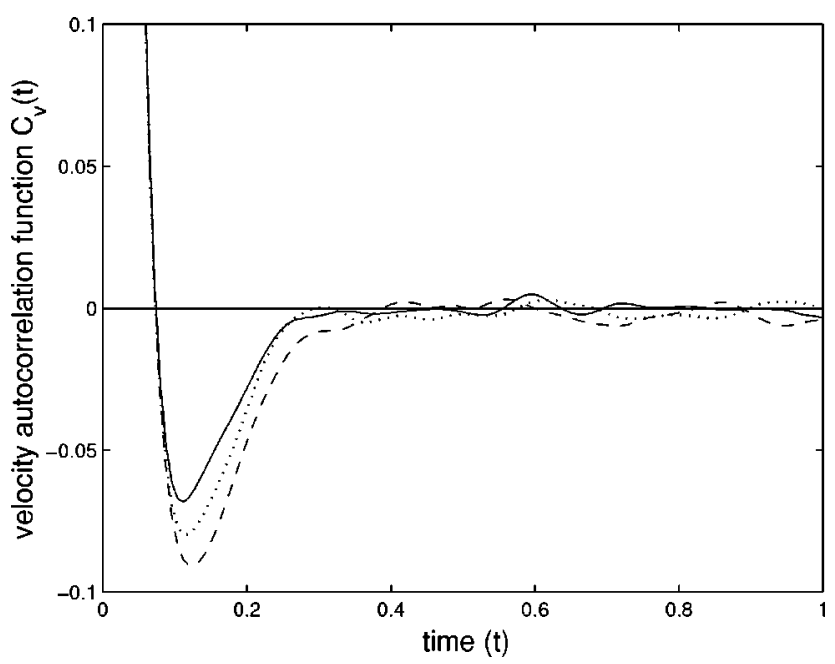

FIG. 9. The velocity autocorrelation function $C_{v}(t)$ for the solute particles at different values of mass ratio $M_{R}$. The solid line represents for $M_{R}$ $=25$, the dotted line for $M_{R}=50$, and the dashed line for $M_{R}=250$. The plot shows an increase in the negative dip with an increase in mass ratio. For details, see the text.

\section{MODE COUPLING THEORY ANALYSIS}

Mode coupling theory remains the only quantitative fully microscopic theory for self-diffusion in strongly correlated systems. In this section we present a mode coupling theory calculation of the solute diffusion for different solvent-to-solute mass ratio $M_{R}$. Diffusion coefficient of a tagged solute is given by the well known Einstein relation,

$$
D_{2}=k_{B} T / m_{2} \zeta_{2}(z=0),
$$

where $D_{2}$ is the diffusion coefficient of the solute, $\zeta_{2}(z)$ is the frequency dependent friction, and $m_{2}$ is the mass of the solute particle. Mode coupling theory provides an expression of the frequency dependent friction on an isolated solute in a solvent.

In the normal liquid regime (in the absence of hopping transport) it can be given by, ${ }^{11,12}$

$$
\frac{1}{\zeta_{2}(z)}=\frac{1}{\zeta_{2}^{B}(z)+R_{21}^{\rho \rho}(z)}+R_{21}^{T T}(z)
$$

where $\zeta_{2}^{B}(z)$ is the binary part of the friction, $R_{21}^{\rho \rho}(z)$ is the friction due to the coupling of the solute motion to the collective density mode of the solvent, and $R_{21}^{T T}(z)$ is the contribution to the diffusion (inverse of friction) from the current modes of the solvent.

For the present system, we have neglected the contribution from the current term, $R_{21}^{T T}(z)$ which is expected to be reasonable at high density and low temperature. Thus the total frequency dependent friction can be approximated as

$$
\zeta_{2}(z) \simeq \zeta_{2}^{B}(z)+R_{21}^{\rho \rho}(z) .
$$

The expression for the time dependent binary friction $\zeta_{2}^{B}(t)$, for the solute-solvent pair, is given by ${ }^{11,12}$

$$
\zeta_{2}^{B}(t)=\omega_{o 12}^{2} \exp \left(-t^{2} / \tau_{\zeta}^{2}\right),
$$

where $\omega_{o 12}$ is now the Einstein frequency of the solute in presence of the solvent and is given by

$$
\omega_{o 12}^{2}=\frac{\rho}{3 m_{2}} \int d \mathbf{r} g_{12}(r) \nabla^{2} v_{12}(r) .
$$

Here $g_{12}(r)$ is the partial solute-solvent radial distribution function.

In Eq. (6), the relaxation time $\tau_{\zeta}$ is determined from the second derivative of $\zeta_{2}^{B}(t)$ at $t=0$ and is given by ${ }^{12,23}$

$$
\begin{aligned}
\omega_{o 12}^{2} / \tau_{\zeta}^{2}= & \left(\rho / 6 m_{2} \mu\right) \int d \mathbf{r}\left(\nabla^{\alpha} \nabla^{\beta} v_{12}(\mathbf{r})\right) g_{12}(\mathbf{r}) \\
& \times\left(\nabla^{\alpha} \nabla^{\beta} v_{12}(\mathbf{r})\right)+(1 / 6 \rho) \\
& \times \int\left[d \mathbf{k} /(2 \pi)^{3}\right] \gamma_{d 12}^{\alpha \beta}(\mathbf{k})(S(k)-1) \gamma_{d 12}^{\alpha \beta}(\mathbf{k}),
\end{aligned}
$$

where summation over repeated indices is implied. $\mu$ is the reduced mass of the solute-solvent pair. Here $S(q)$ is the static structure factor which is obtained from the HMSA scheme. ${ }^{26}$ The expression for $\gamma_{d 12}^{\alpha \beta}(\mathbf{k})$ is written as a combination of the distinct parts of the second moments of the longitudinal and transverse current correlation functions $\gamma_{d 12}^{l}(\mathbf{k})$ and $\gamma_{d 12}^{t}(\mathbf{k})$, respectively, 


$$
\begin{aligned}
\gamma_{d 12}^{\alpha \beta}(\mathbf{k}) & =-\left(\rho / m_{2}\right) \int d \mathbf{r} \exp (-i \mathbf{k} . \mathbf{r}) g_{12}(\mathbf{r}) \nabla^{\alpha} \nabla^{\beta} v_{12}(\mathbf{r}) \\
& =\hat{k}^{\alpha} \hat{k}^{\beta} \gamma_{d 12}^{l}(\mathbf{k})+\left(\delta_{\alpha \beta}-\hat{k}^{\alpha} \hat{k}^{\beta}\right) \gamma_{d 12}^{t}(\mathbf{k}),
\end{aligned}
$$

where $\gamma_{d 12}^{l}(\mathbf{k})=\gamma_{d 12}^{z z}(\mathbf{k})$ and $\gamma_{d 12}^{t}(\mathbf{k})=\gamma_{d 12}^{x x}(\mathbf{k})$.

The expression for $R_{21}^{\rho \rho}(t)$, for the solute-solvent pair, can be written as ${ }^{12,23}$

$$
\begin{aligned}
R_{21}^{\rho \rho}(t)= & \frac{\rho k_{B} T}{m_{2}} \int\left[d \mathbf{k}^{\prime} /(2 \pi)^{3}\right]\left(\hat{k} \cdot \hat{k}^{\prime}\right)^{2} k^{\prime 2}\left[c_{12}\left(k^{\prime}\right)\right]^{2} \\
& \times\left[F^{s}\left(k^{\prime}, t\right) F\left(k^{\prime}, t\right)-F_{o}^{s}\left(k^{\prime}, t\right) F_{o}\left(k^{\prime}, t\right)\right] .
\end{aligned}
$$

In Eq. (10), $c_{12}(k)$ is the two particle (solute-solvent) direct correlation in the wave number $(k)$ space which is obtained here from the HMSA scheme. ${ }^{26}$ The partial radial distribution function $\left[g_{12}(r)\right]$ required to calculate the Einstein frequency $\left(\omega_{o 12}\right)$ and the binary time constant $\left(\tau_{\zeta}\right)$ is obtained from the present simulation study. $F(k, t)$ is the intermediate scattering function of the solvent, and $F_{o}(k, t)$ is the inertial part of the intermediate scattering function. $F_{s}(k, t)$ is the self-intermediate scattering function of the solute and $F_{o}^{s}(k, t)$ is the inertial part of $F_{s}(k, t)$.

It should be noted here that the short time dynamics of the density term used in Eq. (10), is different from the conventional mode coupling formalism. ${ }^{27}$ This prescription has recently been proposed to explain the weak power law mass dependence of the self-diffusion coefficient of a tagged particle. ${ }^{23}$ The detailed discussion on this prescription has been given elsewhere. ${ }^{12,23}$

Since the solvent is much heavier than the solute, the decay of solvent dynamical variables are naturally much slower than those of the solute. Since the decay of $F_{s}(k, t)$ is much faster than $F(k, t)$, in Eq. (10) the contribution from the product, $F_{s}(k, t) F(k, t)$ is mainly governed by the time scale of decay of $F_{s}(k, t)$. Thus the long time part of the $F(k, t)$ becomes unimportant and the viscoelastic expression $^{12}$ for $F(k, z)$ [Laplace transform of $F(k, t)$ ] obtained by using the well-known Mori continued-fraction expansion, truncating at second order would be a reasonably good approximation. The expression of $F(k, z)$, can be written as ${ }^{11,12}$

$$
F(k, z)=\frac{S(k)}{z+\frac{\left\langle\omega_{k}^{2}\right\rangle}{z+\frac{\Delta_{k}}{z+\tau_{k}^{-1}}}},
$$

where $F(k, t)$ is obtained by the Laplace inversion of $F(k, z)$, the dynamic structure factor. Because of the viscoelastic approximation, $\left\langle\omega_{k}^{2}\right\rangle$ and $\Delta_{k}$ and also $\tau_{k}$ are determined by the static pair correlation functions. The static pair correlation functions needed are the static structure factor $S(q)$ and the partial solvent-solvent radial distribution function $g_{11}(r) . S(q)$ is obtained by using the HMSA scheme ${ }^{26}$ and $g_{11}(r)$ is taken from the present simulation study.

We have used the recently proposed ${ }^{12,23}$ generalized selfconsistent scheme to calculate the friction, $\zeta(z)$, which makes use of the well-known Gaussian approximation for $F_{s}(k, t),{ }^{28}$
TABLE III. The contribution of the binary $\left(\zeta_{2}^{B}\right)$ and the density mode $\left(R_{21}^{\rho \rho}\right)$ of the friction for different solvent-to-solute mass ratio $\left(M_{R}\right)$.

\begin{tabular}{ccc}
\hline \hline $\mathbf{M}_{\mathbf{R}}=\frac{\mathbf{m}_{\mathbf{1}}}{\mathbf{m}_{\mathbf{2}}}$ & \multicolumn{3}{c}{$\boldsymbol{\zeta}_{\mathbf{2}}^{\mathbf{B}}$} & $\mathbf{R}_{\mathbf{2 1}}^{\boldsymbol{\rho} \mathbf{}}$ \\
\hline 5 & 23.65 & 13.95 \\
25 & 25.4 & 33.8 \\
50 & 25.45 & 50.2 \\
250 & 25.58 & 99.8 \\
\hline \hline & \\
$F^{s}(k, t)=\exp \left(-\frac{k^{2}\left\langle\Delta r^{2}(t)\right\rangle}{6}\right)$ & \\
$=\exp \left[-\frac{k_{B} T}{m_{2}} k^{2} \int_{0}^{t} d \tau C_{v}(\tau)(t-\tau)\right]$,
\end{tabular}

where $\left\langle\Delta r^{2}(t)\right\rangle$ is the mean square displacement (MSD) and $C_{v}(t)$ is the time-dependent velocity autocorrelation function (VACF) of the solute particles. The time-dependent VACF is obtained by numerically Laplace inverting the frequencydependent VACF, which is in turn related to the frequencydependent friction through the following generalized Einstein relation given by

$$
C_{v}(z)=\frac{k_{B} T}{m_{s}(z+\zeta(z))} .
$$

Thus in this scheme the frequency-dependent friction has been calculated self-consistently with the MSD. The details of implementing this self-consistent scheme is given elsewhere. ${ }^{12,23}$

We have evaluated the diffusion coefficient $D_{2}$ by using the above mentioned self-consistent scheme. The calculated diffusion value was found to be higher than the simulated one. This may be partly due to the observed faster decay of calculated $F_{s}(k, t)$ than the simulated one. This in turn could be due to the Gaussian approximation for $F_{s}(k, t)$ which truncates the cumulant expression of $F_{s}(k, t)$ beyond the quadratic $\left(k^{2}\right)$ term. ${ }^{29}$ However, the higher order terms which are the systematic corrections to the Gaussian forms can be increasingly important at intermediate times and wave numbers $(k) .^{28}$

Therefore, we have performed MCT calculations using the simulated $F_{s}(k, t)$ evaluated at different values of the wave number, $k=n k_{\min }$, where $k_{\min }=2 \pi / \bar{L}$ ( $\bar{L}$ stands for the average size of the simulation cell) and $n$ is an integer varied in the range, $1 \leqslant n \leqslant 35 . F_{s}(k, t)$ is then obtained by interpolating the simulated $F_{s}(k, t)$ so obtained at different wave numbers. The calculated value of the binary term and the density term contribution to the friction for different values of $M_{R}$ are presented in Table III.

Earlier mode coupling theoretical calculations ${ }^{11}$ for small solute was performed by keeping the solute and the solvent mass equal. In those calculations it was found that for size ratio $S_{R}=5$, the solute motion is primarily determined by the binary collision between the solute and the solvent particles. It was also shown that due to the decoupling of the solute motion from the structural relaxation of the solvent, the contribution of the density mode of the sol- 
vent was much smaller than that of the binary term. In the present calculation we find that due to smaller mass of the solute, the contribution of the binary term decreases with an increase in the solvent mass as is clearly evident from Table III.

When compared to the simulated diffusion values, it is clearly seen from Table II that although the MCT qualitatively predicts the diffusion value for $M_{R}=5$, it breaks down at large values of the mass ratios. This may be because MCT overestimates the friction contribution from the density mode. This breakdown of the MCT for large mass ratio can be connected to its breakdown observed near the glass transition temperature. For large solvent mass, the system is almost frozen and the dynamic structure factor of the solvent decays in a much longer time scale when compared to the solute. So from the point of view of the solute, it probes an almost quenched system which can be expected to show the behavior to be very similar to a system, near the glass transition. Just as near the glass transition temperature, the hopping mode also plays the dominant role in the diffusion process.

\section{EFFECT OF DYNAMIC HETEROGENEITY ON $F_{s}(k, t)$ OF THE SOLUTE}

It is well-known that the self-intermediate scattering function, $F_{s}(k, t)$ can be formally expressed by the following cumulant expansion in powers of $k^{2}:{ }^{29}$

$$
\begin{aligned}
F_{s}(k, t)= & \exp \left(-\frac{1}{6} k^{2}\left\langle\Delta r^{2}(t)\right\rangle\right)\left[1+\frac{1}{2} \alpha_{2}(t)\right. \\
& \left.\times\left(\frac{1}{6} k^{2}\left\langle\Delta r^{2}(t)\right\rangle\right)^{2}+O\left(k^{6}\right)\right],
\end{aligned}
$$

where $\alpha_{2}(t)$ is defined as

$$
\alpha_{2}(t)=\frac{3\left\langle\Delta r^{4}(t)\right\rangle}{5\left\langle\Delta r^{2}(t)\right\rangle^{2}}-1 .
$$

In the stable fluid range, it has been generally found that the Gaussian approximation to $F_{s}(k, t)$, the leading term in the above cumulant expansion, provides a reasonably good description of the dynamics of the system. The higher order terms which are the systematic corrections to the Gaussian approximation in the cumulant expansion are found to be small.

However, in a supercooled liquid, this is not the case. The dynamical heterogeneities observed in a deeply supercooled liquid are often manifested as the magnitude of the deviation of $\alpha_{2}(t)$, the so-called non-Gaussian parameter, from zero. It has been observed that $\alpha_{2}(t)$ deviates more and more strongly and decays more and more slowly with an increase in the degree of supercooling. ${ }^{30}$ In the present study, a similar behavior has also been observed in $\alpha_{2}(t)$ (calculated for the solute). The height of the maximum in $\alpha_{2}(t)$ increases as the mass of the solvent is increased (see Fig. 5), which is clear evidence that the solute probes increasingly heterogeneous dynamics.

It is generally believed that the dominant corrections to the Gaussian result are provided by the term containing $\alpha_{2}(t)$. Thus, it would be interesting to see whether this term alone is sufficient to explain the observed stretching in

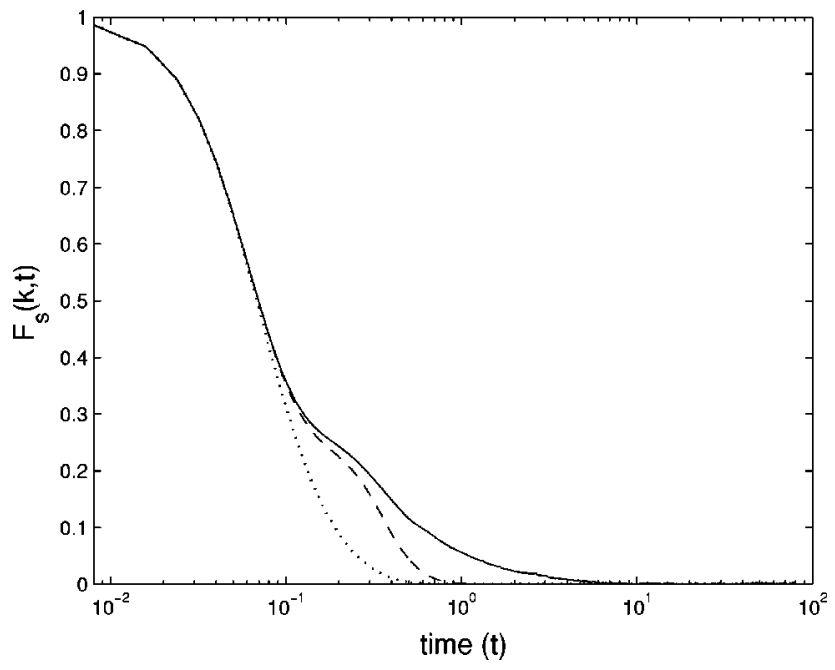

FIG. 10. Comparison of the simulated self-intermediate scattering function $F_{s}(k, t)$ of the solute particles with the $F_{s}(k, t)$ obtained after incorporating the lowest order correction $\left(k^{4}\right.$ term) to the Gaussian approximation in the cumulant expansion [Eq. (14)]. The Gaussian approximation to $F_{s}(k, t)$ is also shown. The mean-squared displacement $\left[\left\langle\Delta r(t)^{2}\right\rangle\right]$ and the nonGaussian parameter $\left[\alpha_{2}(t)\right]$ required as an input are obtained from the simulation. The plot is at the reduced wave number $k^{*} \sim 2 \pi / \sigma_{12}$ and for the mass ratio $M_{R}=50 . F_{s}(k, t)$ obtained from the simulation is represented by the solid line, the dashed line represents the $F_{s}(k, t)$ obtained after the lowest order correction to the Gaussian approximation, and the dotted line represents the Gaussian approximation. For a detailed discussion, see the text.

$F_{s}(k, t)$ of the solute at a longer time. In order to quantify this, we have plotted in Fig. 10 the simulated $F_{s}(k, t)$ along with the $F_{s}(k, t)$ obtained after incorporating the lowest order correction $\left(k^{4}\right.$ term) to the Gaussian approximations for mass ratio, $M_{R}=50$ at the reduced wave number $k^{*}$ $\sim 2 \pi / \sigma_{12}$. For comparison, the Gaussian approximation to $F_{s}(k, t)$ is also shown. It is clearly seen that the first nonGaussian correction to $F_{s}(k, t)$ is not sufficient to describe the long time stretching predicted by the simulation. This clearly indicates that at the length scales probed by the solute, the higher order corrections cannot be neglected. It is the nearly quenched inhomogeneity probed by the solute particles over small length scales which play an important role in the dynamics of the system. The stretching of $F_{s}(k, t)$ observed in simulation could be intimately connected with this nearly quenched inhomogeneity probed by the solute particles.

\section{CONCLUSIONS}

Let us first summarize the main results of this study. We have investigated by using the molecular dynamics simulation the diffusion of small light particles in a solvent composed of larger massive particles for a fixed solvent-to-solute size ratio $\left(S_{R}=5\right)$ but with a large variation in mass ratio (where the mass of the solute is kept constant). In addition, a mode-coupling theory (MCT) analysis of diffusion is also presented. It is found that the solute dynamics remain surprisingly coupled to the solvent dynamics even in the limit of highly massive solvent. Most interestingly, with increase in mass ratio, the self-intermediate scattering function of the solute develops a stretching at long time which, for interme- 
diate values of mass ratio, could be fitted to a single stretched exponential function with the stretching exponent, $\beta \simeq 0$.6. In the limit of very large mass ratio, the existence of two stretched exponential separated by a power law type plateau is observed. This behavior is found to arise from increasingly heterogeneous environment probed by the solute particle as one increases the mass of the solvent particles. The MCT calculation of self-diffusion is found to agree qualitatively with the simulation results for small mass ratio. However, it fails to describe the simulated prediction at large mass ratios. The velocity correlation function of the solute shown interesting oscillatory structure.

Several of the results observed here are reminiscent of the relaxation of the self-intermediate scattering function, $F_{s}(k, t)$ observed in the deeply supercooled liquid near its glass transition temperature. In that case also, one often observes a combination of power-law and stretched exponential in the decay of the intermediate scattering function. We find that even the breakdown of MCT at large mass ratio could be connected to its breakdown near the glass transition temperature because it is the neglect of the spatial hopping mode of particles which is responsible for the breakdown of MCT. It is to be noted that those hoppings which are mostly ballistic in nature (after a binary collision) have already been incorporated in MCT. However, MCT does not include the hoppings which involve collective displacement involving several molecules. ${ }^{15}$

It should be pointed out that in the MCT calculation, we have neglected the contribution of the current term. While the current contribution may improve the agreement between the simulation and MCT result for small mass ratio $\left(M_{R}\right.$ $=5$ ), its contribution at larger mass ratio is not expected to change the results significantly, because the discrepancy is very large.

The origin of the power law remains to be investigated in more detail. Our preliminary analysis shows that this may be due to the separation of the time scale between the first weakly stretched exponential (due to the dispersion in the binary-type interaction term) and the second, later more strongly stretched exponential (which is due to the coupling of the solute's motion to the density mode of the slow solvent). This separation arises because these two motions are very different in nature. However, a quantitative theory of this stretching and power-law is not available at present.

While the origin of the stretching of $F_{s}(k, t)$ can be at least qualitatively understood in terms of the inhomogeneity experienced by the solute, the origin of hopping is less clear. In the supercooled liquid, hopping is found to be correlated with an anisotropic local stress ${ }^{15}$ which is unlikely in the present system which is at lower density and pressure.

Finally, we note that the system investigated here is a good candidate to understand qualitative features of relaxation in a large variety of systems, such as, concentrated solution of polysaccharide in water and also the motion of water in clay.

\section{ACKNOWLEDGMENTS}

This work was supported in part by the Council of Scientific and Industrial Research (CSIR), India and the Department of Science and Technology (DST), India. One of the authors (R.K.M.) thanks the University Grants Commission (UGC) for providing the Research Scholarship.

${ }^{1}$ L. A. Bunimovich and Ya. G. Sinai, Commun. Math. Phys. 78, 247 (1980); 78, 479 (1980).

${ }^{2}$ J. Machta and R. Zwanzig, Phys. Rev. Lett. 50, 1959 (1983); R. Zwanzig, J. Stat. Phys. 30, 275 (1983).

${ }^{3}$ B. Bagchi, R. Zwanzig, and M. C. Marchetti, Phys. Rev. A 31, 892 (1985).

${ }^{4}$ R. Zwanzig and A. K. Harrison, J. Chem. Phys. 83, 5861 (1985).

${ }^{5}$ G. Phillies, J. Phys. Chem. 85, 2838 (1981).

${ }^{6}$ G. L. Pollack and J. J. Enyeart, Phys. Rev. A 31, 980 (1985); G. L. Pollack, R. P. Kennan, J. F. Himm, and D. R. Stump, J. Chem. Phys. 92, 625 (1990).

${ }^{7}$ B. A. Kowert, K. T. Sobush, N. C. Dang, L. G. Seele III, C. F. Fuqua, and C. L. Mapes, Chem. Phys. Lett. 353, 95 (2002).

${ }^{8}$ F. Ould-Kaddour and J.-L. Barrat, Phys. Rev. A 45, 2308 (1992).

${ }^{9}$ F. Ould-Kaddour and D. Levesque, Phys. Rev. E 63, 011205 (2000).

${ }^{10}$ A. J. Easteal and L. A. Woolf, Chem. Phys. Lett. 167, 329 (1990).

${ }^{11}$ S. Bhattacharyya and B. Bagchi, J. Chem. Phys. 106, 1757 (1997).

${ }^{12}$ B. Bagchi and S. Bhattacharyya, Adv. Chem. Phys. 116, 67 (2001).

${ }^{13}$ R. Castillo, C. Garza, and S. Ramos, J. Phys. Chem. 98, 4188 (1994).

${ }^{14}$ P. B. Conrad and J. J. de Pablo, J. Phys. Chem. A 103, 4049 (1999); N. C. Ekdawi-Sever, P. B. Conrad, and J. J. de Pablo, ibid. 105, 734 (2001)

${ }^{15}$ S. Bhattacharyya and B. Bagchi, Phys. Rev. Lett. 89, 025504 (2002).

${ }^{16}$ M. P. Allen and D. J. Tildesley, Computer Simulation of Liquids (Oxford University Press, Oxford, 1987).

${ }^{17}$ G. J. Martyna, D. J. Tobias, and M. L. Klein, J. Chem. Phys. 101, 4177 (1994); H. C. Andersen, ibid. 72, 2384 (1980); S. Nose, Mol. Phys. 52, 255 (1984); W. G. Hoover, Phys. Rev. A 31, 1695 (1985).

${ }^{18}$ G. J. Martyna, M. E. Tuckerman, and M. L. Klein, J. Chem. Phys. 97, 2635 (1992)

${ }^{19}$ M. E. Tuckerman, G. J. Martyna, and B. J. Berne, J. Chem. Phys. 97, 1990 (1992).

${ }^{20}$ H. Yoshida, Phys. Lett. A 150, 260 (1990); M. Suzuki, J. Math. Phys. 32, 400 (1991).

${ }^{21}$ G. J. Martyna, M. E. Tuckerman, D. J. Tobias, and M. L. Klein, Mol. Phys. 87, 1117 (1996).

${ }^{22}$ A. Rahman, Phys. Rev. A 136, 405 (1964).

${ }^{23}$ S. Bhattacharyya and B. Bagchi, Phys. Rev. E 61, 3850 (2000).

${ }^{24}$ B. Bernu, J. P. Hansen, Y. Hiwatari, and G. Pastore, Phys. Rev. A 36, 4891 (1987); D. Thirumalai and R. D. Mountain, J. Phys. C 20, L399 (1987).

${ }^{25}$ J. E. Variyar, D. Kivelson, and R. M. Lynden-Bell, J. Chem. Phys. 97, 8549 (1992)

${ }^{26}$ S. A. Egorov, M. D. Stephens, A. Yethiraj, and J. L. Skinner, Mol. Phys. 88, 477 (1996).

${ }^{27}$ L. Sjogren and A. Sjolander, J. Phys. C 12, 4369 (1979).

${ }^{28} \mathrm{U}$. Balucani and M. Zoppi, Dynamics of the Liquid State (Oxford University Press, New York, 1994).

${ }^{29}$ B. R. A. Nijboer and A. Rahman, Physica (Amsterdam) 32, 415 (1966).

${ }^{30}$ W. Kob, C. Donati, S. J. Plimpton, P. H. Poole, and S. C. Glotzer, Phys. Rev. Lett. 79, 2827 (1997). 